OPEN ACCESS

Edited by:

Tomomi Watanabe-Asaka,

Tohoku Medical and Pharmaceutical

University, Japan

Reviewed by:

Amir Sagi,

Ben-Gurion University of the Negev,

Israel

Hidekazu Katayama,

Tokai University, Japan

*Correspondence:

Haihui Ye

hhye@jmu.edu.cn

Specialty section:

This article was submitted to

Aquatic Physiology,

a section of the journal

Frontiers in Marine Science

Received: 11 February 2021

Accepted: 16 March 2021

Published: 06 April 2021

Citation:

Liu F, Shi W, Ye H, Liu A and Zhu Z (2021) RNAi Reveals Role

of Insulin-Like Androgenic Gland

Hormone 2 (IAG2) in Sexual

Differentiation and Growth in

Hermaphrodite Shrimp.

Front. Mar. Sci. 8:666763.

doi: 10.3389/fmars.2021.666763

\section{RNAi Reveals Role of Insulin-Like Androgenic Gland Hormone 2 (IAG2) in Sexual Differentiation and Growth in Hermaphrodite Shrimp}

\author{
Fang Liu', Wenyuan Shi', Haihui Ye ${ }^{2 *}$, An Liu' ${ }^{2}$ and Zhihuang Zhu ${ }^{3}$ \\ ${ }^{1}$ College of Ocean and Earth Sciences, Xiamen University, Xiamen, China, ${ }^{2}$ College of Fisheries, Jimei University, Xiamen, \\ China, ${ }^{3}$ Fisheries Research Institute of Fujian, Xiamen, China
}

Insulin-like androgenic gland hormone (IAG) is the most widely known hormone that regulates sexual differentiation in crustaceans. Previously, a transcriptome study described two transcripts of IAGs (Lvit-IAG1 and Lvit-IAG2) in the peppermint shrimp Lysmata vittata, a species characterized by a rare reproductive system of protandric simultaneous hermaphroditism (PSH). Herein, we explored the function of Lvit-IAG2 via RNA interference (RNAi) experiments, and then compared the functional differences between the two IAGs. We demonstrated that Lvit-IAG2 positively regulated the expression of crustacean hyperglycemic hormone ( $(\mathrm{vit}-\mathrm{CHH})$ in eyestalk ganglion but exhibited no significant effect on the expression of gonad-inhibiting hormone ( $\mathrm{V} v \mathrm{t}-\mathrm{G} / \mathrm{HS}$ ) and crustacean female sex hormone (Lvit-CFSHS). Besides, Lvit-IAG2 gene knockdown caused degeneration in appendices masculinae (AM) and suppressed germ cells at the secondary spermatocyte stage. Moreover, silencing the Lvit-IAG2 gene impeded ovarian development, including smaller previtellogenic oocytes, and lower expression of vitellogenin ( $L v i t-V g$ ) gene in hepatopancreas and vitellogenin receptor ( $L$ vit-VgR) gene in the ovarian region. Notably, silencing the Lvit-IAG2 gene impeded individual growth of the species. Collectively, findings from this study demonstrate that Lvit-IAG2 and LvitIAG1 coordinatively function to modulate sexual differentiation in L. vittata; meanwhile, Lvit-IAG2 stimulates the growth of the PSH species.

Keywords: sexual differentiation, IAG, PSH, reproductive endocrine, crustacean

\section{INTRODUCTION}

Gonochorism is the most common reproductive strategy in decapod crustacean species (Juchault, 1999). Males and females have significantly different reproductive systems, as well as external features (Nagaraju, 2011). For instance, the reproductive system of male American lobster Homarus americanus consists of the paired testes, vas deferens, spermiducts, and gonopores located on the fifth pair of walking legs. Besides, the specialized first pair of pleopods serves as a gonopod during mating (Comeau and Benhalima, 2018b). However, the female reproductive system of $H$. americanus consists of the paired ovaries, oviducts, and gonopores located on the third pair of walking legs. The specialized organ, seminal receptacle, arises on the thoracic ventral side between the fourth and the fifth pairs of walking legs (Comeau and Benhalima, 2018a). 
Interestingly, a review of the literature shows that Caridean shrimps exhibit several other protandry sexual systems apart from gonochorism (Bauer, 2000). Among them, strictly sequential protandric hermaphroditism (SPH) and protandric simultaneous hermaphroditism (PSH) are well-studied. For example, Northern spot shrimp Pandalus platyceros, a $\mathrm{SPH}$ species, first mature as functional males with hermaphrodite gonads, and then go to a transitional phase followed by a functional female phase (Levy et al., 2020b). Meanwhile, all the Lysmata species with the PSH sexual system, eventually acquire reproductive functions for both males and females following the male phase and a short transitional phase (Bauer, 2000; Chen et al., 2019).

As with insect, sexual differentiation is controlled by key reproductive hormones in decapods. It is generally believed that insulin-like androgenic gland hormone (IAG) regulates male sexual differentiation in dioecious decapods (Ventura et al., 2009). In decapod species, IAG was first identified in the androgenic gland (AG) of the red-claw crayfish Cherax quadricarinatus in 2007 (Manor et al., 2007). Since then, numerous studies have found IAG in dioecious (Ventura et al., 2009; Rosen et al., 2010; Ventura et al., 2012; Chung, 2014; Huang et al., 2014), parthenogenetic (Levy et al., 2017), and hermaphroditic (Zhang et al., 2017; Levy et al., 2020a; Liu et al., 2020) species. Furthermore, its critical role in sexual differentiation has been properly studied via loss-of-function experiments (Ventura et al., 2012; Zmora and Chung, 2014; Priyadarshi et al., 2017; Fu et al., 2020). For instance, in the giant freshwater prawn, Macrobrachium rosenbergii, IAG gene silencing induced degeneration of male primary and secondary characteristics (Ventura et al., 2009), and feminization in young males (Ventura et al., 2012). Silencing IAG gene could also feminize male-related phenotypes in the intersex red-claw crayfish C. quadricarinatus (Rosen et al., 2010) and the male Chinese mitten crab Eriocheir sinensis (Fu et al., 2020). Moreover, augmentation of IAG hormone transcripts in adult $M$. rosenbergii males resulted in significantly higher (orange claw) OC to (blue claw) BC transformations and vice versa (Priyadarshi et al., 2017). Considering its universal and pivotal role as a master regulator of crustacean sexual development, IAG is regard as the sexual "IAG-switch" in decapod crustacean (Levy and Sagi, 2020).

Crustacean female sex hormone (CFSH) is a neurohormone that is closely related to female sexual differentiation in decapods. It was first isolated from the eyestalk ganglion in the Atlantic blue crab Callinectes sapidus, and was found to be pivotal in the development of the female's mating and egg brooding systems, including the gonopores and ovigerous setae (Zmora and Chung, 2014). Similar functions have also been established in the mud crab Scylla paramamosain (Jiang et al., 2020). Besides, CFSH was also reported as an inhibitor of IAG, as recombinant $S p$ $\mathrm{CFSH}$ protein significantly suppressed $S p-I A G$ expression in the AG explants of the mud crab $S$. paramamosain in vitro (Liu et al., 2017).

In crustacean males, previous studies have revealed that the $\mathrm{X}$-organ-sinus gland complex (XO-SG) in the eyestalk ganglion, a major source of neuropeptides, modulates reproductive functions via the eyestalk-AG-testicular axis (Khalaila et al., 2002;
Nagaraju, 2011). Besides, its inhibitory effects on AG development and IAG expression have been intensively explored through eyestalk ablation. The active components suppressing AG development have been identified in XO-SG. Among them, researchers have comprehensively characterized the crustacean hyperglycemic hormone $(\mathrm{CHH})$ superfamily neuropeptides as well as CFSH mentioned above. Most members of the $\mathrm{CHH}$ superfamily exerted inhibitory effects on either AG development or IAG expression (Das et al., 2015; Li et al., 2015; Guo et al., 2019). Following the silencing of gonad-inhibiting hormone $(\mathrm{GIH})$, molt-inhibiting hormone $(\mathrm{MIH})$, and $\mathrm{CHH}$ gene, $I A G$ transcription level increased significantly in the black tiger prawn Penaeus monodon (Das et al., 2015), the oriental river prawn Macrobrachium nipponense (Li et al., 2015), and the Pacific white shrimp Penaeus (Litopenaeus) vannamei (Guo et al., 2019).

To date, however, there are rare reports elaborating sexual differentiation mechanism mediated by IAG in protandric crustaceans. In the SPH shrimp P. platyceros, Pnp-IAG knockdown elevated vitellogenin gene expression in the hepatopancreas and transformation of the gonad from ovotestis to ovary (Levy et al., 2020a). Zhang et al. (2017) identified an IAG transcript $(L w-I A G)$ in a PSH shrimp Lysmata wurdemanni, and examined its expression profile during gonadal development. The very low but detectable expression of $L w$-IAG during the euhermaphrodite phases suggested that $L w-I A G$ was possibly responsible for the maintenance of the male reproductive activity in euhermaphrodite phase. Recently, a transcriptomic study identified two insulin-like peptide (ILP) transcripts (Lvit-IAG1 and Lvit-IAG2) in the peppermint shrimp Lysmata vittata (Bao et al., 2020), and biological functions of Lvit-IAG1 have been explored in detail (Liu et al., 2020). In L.vittata, silencing Lvit-IAG1 impeded development of male-related phenotypes (appendices masculinae (AM) and male gonopores) while suppressing the germ cells at the primary spermatocyte stage, demonstrating that Lvit-IAG1 was indeed a functional IAG. Meanwhile, Lvit-IAG1 was also suggested to regulate the ovarian development by inhibiting Lvit-GIHs and Lvit-CFSHs expression in the eyestalk ganglion (Liu et al., 2020). In decapods, four types of insulin and related peptides were identified: insulinlike androgenic gland hormone (IAG), insulin, relaxins, and gonadulins (Veenstra, 2020). Besides, there is usually only one IAG gene identified in a species ( $\mathrm{Li}$ et al., 2012; Huang et al., 2017b). Therefore, further in-depth studies are warranted to explore whether Lvit-IAG2 is another functional IAG or other insulin and related peptides.

The peppermint shrimp L. vittata, a relatively small caridean species (maximum carapace length $<1 \mathrm{~cm}$ ), like other species, displays a unique sexual system, PSH, whereby individuals first mature as males; however, with increasing age and size, they acquire reproductive functions for both males and females (Bauer, 2000; Alves et al., 2019). Given its impressive reproductive fecundity, short generation time, and relatively clear ontogenetic gonad development staging system, L. vittata is a good model organism for exploring the molecular mechanisms for endocrine regulation of the unique PSH sexual system in crustaceans (Chen et al., 2019). 
In this study, we continuously explored the putative function of Lvit-IAG2 in L. vittata. Following its similar spatial and temporal expression profiles with Lvit-IAG1, we hypothesized that Lvit-IAG2 and Lvit-IAG1 exert similar biological functions and they coordinatively regulate sexual differentiation of the species. To validate this hypothesis, we performed both short-term and long-term gene knockdown via RNA interference (RNAi). In addition to morphological characteristics, expression levels of gonadal developmentassociated genes $[G I H$, vitellogenin $(\mathrm{Vg})$, and vitellogenin Receptor $(\operatorname{VgR})]$, carbohydrate metabolism $(\mathrm{CHH})$, and sexual differentiation $(C F S H)$ were assessed via qRT-PCR.

\section{MATERIALS AND METHODS}

\section{Animals}

The experimental animals (L. vittata) were captive-bred at the Fisheries Research Institute of Fujian Province in Xiamen city, China. They were then acclimated in seawater aquaria for 2 days under these conditions: temperature of $25.5 \pm 0.5^{\circ} \mathrm{C}$ and salinity of $32 \pm 1$ PSU. During this period, the animals were fed on a commercially formulated shrimp diet daily. A recent study from this laboratory had described two developmental phases covering four gonadal development stages for L. vittata, which we followed to define the gonadal stages in the present experiment. Three gonadal development stages (Stage I: ovarian region is smaller than testicular region and both regions are transparent; Stage II: both regions are cloudy white and they are of similar size; and Stage III: ovarian region becomes earth brown and bigger than the testicular region while testicular region is still cloudy white) are included in the male phase, during which the testicular part of the gonad becomes sequentially mature while the ovarian part is still immature. Both testicular region and ovarian region are fully developed and filled with mature germ cells in the euhermaphrodite phase (Stage IV), during which individuals acquire reproductive functions for both males and females (Chen et al., 2019). Animal handling and experimental procedures were performed with strict adherence to the guidelines approved by the Xiamen University Animal Care and Use Committee.

\section{Fragment Cloning of Lvit-IAG2}

The total RNA was extracted from the androgenic gland at gonadal development stage I using HiPure Universal RNA Kit (Magen) following protocol stipulated by the manufacturer. The first-strand cDNA was generated from $1 \mu \mathrm{g}$ total RNA using RevertAid First Strand cDNA Synthesis Kit (Fermentas). To verify the accuracy of the predicted open reading frame (ORF) polymerase chain reaction (PCR) was performed by specific primers to ascertain its suitability for the subsequent studies. The PCR reaction was prepared with Ex-Taq polymerase (TaKaRa) and run under the following conditions: $95^{\circ} \mathrm{C}$ for $3 \mathrm{~min} ; 35$ cycles of $95^{\circ} \mathrm{C}$ for $30 \mathrm{~s}, 60^{\circ} \mathrm{C}$ for $30 \mathrm{~s}$, and $72^{\circ} \mathrm{C}$ for $30 \mathrm{~s}$, followed by $72^{\circ} \mathrm{C}$ for $5 \mathrm{~min}$ final extension. The PCR products were visually examined in $1.0 \%$ agarose gel. Subsequently, we purified LvitIAG2 fragments and inserted them into the pMD19-T vector
(TaKaRa) for sequencing. Specific primers used are listed in Table 1.

\section{Bioinformatics Analyses}

The primers used for fragment cloning and dsRNA preparation were designed via the Primer 5.0 software. Then we adopted the ORF Finder software ${ }^{1}$ to predict the open reading frame (ORF), whereas the SignalP-5.0 Server ${ }^{2}$ was used to predict the signal peptides. Further, cysteine residues and putative disulfide bonds were predicted via the DiANNA 1.1 web server ${ }^{3}$. To align the deduced amino acid sequences with reported sequences, we used the Clustal Omega website ${ }^{4}$. The N-glycosylation motif was predicted by the NetNGlyc 1.0 Server $^{5}$.

The Maximum Likelihood method with 1,000 bootstrap replicates based on the JTT matrix-based model in MEGA7 was applied to generate a phylogenetic tree entailing the deduced amino acid sequence alignments and excluding signal peptide of insulin-related peptides. IAG sequences were shown in Table 2.

\footnotetext{
${ }^{1}$ https://www.ncbi.nlm.nih.gov/orffinder/

${ }^{2}$ http://www.cbs.dtu.dk/services/SignalP/

${ }^{3}$ http://clavius.bc.edu/ clotelab/DiANNA/

${ }^{4}$ https://www.ebi.ac.uk/Tools/msa/clustalo/

${ }^{5}$ http://www.cbs.dtu.dk/services/NetNGlyc/
}

TABLE 1 | Summary of primers used in this study.

\begin{tabular}{|c|c|c|}
\hline Primer & Sequence $\left(5^{\prime}-3^{\prime}\right)$ & Application \\
\hline IAG2F & TAACCAAGAAATTCACCGTGAAAATGG & Fragment validation \\
\hline IAG2R & ACGCTGTAGTCAAGCCATTGGACC & \\
\hline CFSH1QF & ATCCACACCTCAGAACTCATC & RT-PCR/qRT-PCR \\
\hline CFSH1QR & GCACAGGCTACGGTTATCT & \\
\hline CFSH2QF & CAAGGACGGCGATGATGA & \\
\hline CFSH2QR & GCGAAGGATCTGAGATGTGTA & \\
\hline IAG1QF & СTAATCTTGCTGCСTCATTCTAC & \\
\hline IAG1QR & GCGTCGTTCTCTGTAATAATCG & \\
\hline IAG2QF & TCAGTCTCAGCCATCTCCT & \\
\hline IAG2QR & TGAACCGACCACCTCTAATG & \\
\hline $\mathrm{CHHQF}$ & CATCTATGACCGTGAACTCTT & \\
\hline $\mathrm{CHHQF}$ & TACTTGCCGACCATCTGA & \\
\hline GIH1QF & GACTTCCTGTGGTGCGTGTA & \\
\hline GIH1QR & GCTCGCAGTATGCTCATGGA & \\
\hline GIH2QF & ATATGGCGTGTGGTTCTG & \\
\hline GIH2QR & GAAGTGAGCGGACTACATT & \\
\hline VgQF & GCAAAAGTGGGAGCCGAAAG & \\
\hline $\operatorname{VgQR}$ & ATCACCCGTAGAGGGTAGGG & \\
\hline VgRQF & CTGCGTCTCGGAACTCAA & \\
\hline VgRQR & GTGCTGGTGGTGAAGATGA & \\
\hline actinF & CGTGACCTGACTGATTACC & \\
\hline actinR & CGTTACCGATAGTGATTACCT & \\
\hline IAG2dsF & CTCTGTAAATCAGTCTCAGCCATCT & dsRNA synthesis \\
\hline IAG2dsR & ATACCGTCTTGCAGAATTTCACA & \\
\hline GFPdsF & TGGGCGTGGATAGCGGTTG & \\
\hline GFPdsR & GGTCGGGGTAGCGGCTGAAG & \\
\hline T7primer & TAATACGACTCACTATAGGG & \\
\hline SP6primer & ATTAGGTGACACTATAG & \\
\hline
\end{tabular}


TABLE 2 | Summary of IAG sequences used in multiple sequence alignment and phylogenetic analysis.

\begin{tabular}{lll}
\hline Sequence & Species & $\begin{array}{l}\text { GenBank accession } \\
\text { number }\end{array}$ \\
\hline Pch-IAG1 & Penaeus chinensis & AFU60548.1 \\
Pch-IAG2 & Penaeus chinensis & AFU60549.1 \\
Pm-IAG & Penaeus monodon & ADA67878.1 \\
Pv-IAG & Penaeus vannamei & AlR09497.1 \\
Pj-IAG & Penaeus japonicus & BAK20460.1 \\
Je-IAG & Jasus edwardsii & AIM55892.1 \\
Sv-IAG & Sagmariasus verreauxi & AHY99679.1 \\
Cd-IAG & Cherax destructor & ACD91988.1 \\
Cqua-IAG & Cherax quadricarinatus & ABH07705.1 \\
Pc-IAG & Procambarus clarkii & ALX72789.1 \\
Pf-IAG & Procambarus fallax & ASM94213.1 \\
Lvit-IAG1 & Lysmata vittata & MT114196 \\
Lvit-IAG2 & Lysmata vittata & MT114197 \\
Es-IAG & Eriocheir sinensis & AVK43106.1 \\
Cqui-IAG & Chaceon quinquedens & ASA45642.1 \\
Sp-IAG & Scylla paramamosain & AFY09905.1 \\
Cs-IAG & Callinectes sapidus & AEI72263.1 \\
Pp-IAG & Pandalus platyceros & ASM94212.1 \\
Ppac-IAG & Palaemon pacificus & BAJ84109.1 \\
Ppau-IAG & Palaemon paucidens & BAJ84108.1 \\
MI-IAG & Macrobrachium lar & BAJ78349.1 \\
Mv-IAG & Macrobrachium vollenhovenii & AHZ34725.1 \\
Lw-IAG & Lysmata wurdemanni & $10.1371 /$ journal. \\
& & pone.0172782 (Zhang \\
Mn-IAG & Macrobrachium nipponense & AHA33389.1 \\
Mr-IAG & Macrobrachium rosenbergii & AWU67706.1 \\
\hline & & \\
& &
\end{tabular}

Insulin, relaxins, and gonadulins sequences were borrowed from previous works by Veenstra (2020).

\section{The qRT-PCR Assays}

Primers used for quantitative real-time PCR (qRT-PCR) were designed by the Beacon Designer 8 software. RT-PCR products were sequenced as described in section "Fragment cloning of Lvit-IAG2" for accuracy. The melting curves were subjected to intensive analysis to ensure primer specificity. A standard curve was used to calculate the amplification efficiency of each primer pair. Notably, each selected primer pair exhibited a suitable PCR amplification efficiency (96.3-104.5\%, see Table 3). The first-strand cDNA was generated from 300 ng total RNA using TransScript ${ }^{\circledR}$ II One-Step gDNA Removal and cDNA short SuperMix Kit (TransGen). The cDNA was diluted to four folds using RNase-free water before it was utilized in qRT-PCR detection. Components including, $10 \mu \mathrm{l}$ TB Green Premix Ex Taq II (2X) (TaKaRa), $2 \mu$ l diluted cDNA, $0.5 \mu l$ forward/reverse primer $(1 \mathrm{mM})$, and $7 \mu \mathrm{l}$ RNase-free water, were used for a $20 \mu \mathrm{l}$ qRT-PCR reaction system. The reaction was performed using 7500 Real-Time PCR (Applied Biosystems) with the following steps: $95^{\circ} \mathrm{C}$ for $30 \mathrm{~s}$, followed by 40 cycles of $95^{\circ} \mathrm{C}$ for $15 \mathrm{~s}$, $58.5^{\circ} \mathrm{C}$ for $15 \mathrm{~s}$, and $72^{\circ} \mathrm{C}$ for $30 \mathrm{~s}$. The result was calculated using
TABLE 3 | Summary of qPCR efficiency of the primer pairs.

\begin{tabular}{llcc}
\hline Gene & Primer pairs & $\begin{array}{c}\text { Annealing } \\
\text { temperature }\left({ }^{\circ} \mathbf{C}\right)\end{array}$ & $\begin{array}{c}\text { Amplification } \\
\text { efficiency (\%) }\end{array}$ \\
\hline Lvit-IAG1 & IAG1QF/AG1QR & 58.5 & 98.9 \\
Lvit-IAG2 & IAG2QF/IAG2QR & 58.5 & 104.5 \\
Lvit-CFSH1 & CFSH1QF/CFSH1QR & 58.5 & 99.1 \\
Lvit-CFSH2 & CFSH2QF/CFSH2QR & 58.5 & 103.2 \\
Lvit-GIH1 & GIH1QF/GIH1QR & 58.5 & 98.8 \\
Lvit-GIH2 & GIH2QF/GIH2QR & 58.5 & 98.5 \\
Lvit-CHH & CHHQF/CHHQR & 58.5 & 101.7 \\
Lvit-Vg & VgQFNgQR & 58.5 & 96.3 \\
Lvit-VgR & VgRQF/NgRQR & 58.5 & 101.8 \\
Lvit- $\beta-a c t i n$ & actinQF/actinQR & 58.5 & 101.2 \\
\hline
\end{tabular}

the $2^{-\Delta \Delta C t}$ method, Lvit- $\beta$-actin (GenBank accession number: MT114194) as the reference gene.

\section{Tissue Expression Profile of Lvit-IAG2 in L. vittata}

As described in section "Fragment cloning of Lvit-IAG2," total RNA was extracted from various tissues (eyestalk ganglion, brain, thoracic ganglion, abdominal ganglion, ovary, testis, AG, hepatopancreas, stomach, intestine, heart, gill, and muscle). The first-strand cDNA synthesis was performed as described in section "The qRT-PCR assays." RT-PCR tissue expression profile detection was conducted under the following conditions: $95^{\circ} \mathrm{C}$ for $3 \mathrm{~min} ; 35 \mathrm{cycles}$ of $95^{\circ} \mathrm{C}$ for $30 \mathrm{~s}, 58.5^{\circ} \mathrm{C}$ for $30 \mathrm{~s}$ and $72^{\circ} \mathrm{C}$ for $30 \mathrm{~s}$, followed by $72^{\circ} \mathrm{C}$ for $5 \mathrm{~min}$ final extension. Meanwhile, Lvit- $\beta$-actin was amplified as a positive control, with similar PCR conditions as described above. RT-PCR products were examined using $1.5 \%$ agarose gel, then images taken by a UV detector (Geldoc, Thermo Fisher Scientific).

\section{Expression Profiles of Lvit-IAG2 and Lvit-IAG1 During Gonadal Development}

The AGs of $L$. vittata at different gonadal development stages (IIV) $(n=5)$ were obtained. This was followed by RNA extraction, the first-strand cDNA synthesis, and qRT-PCR analysis as described in sections "Fragment cloning of Lvit-IAG2" and "The qRT-PCR assays.”

\section{dsRNA Preparation}

Lvit-IAG2 fragment encoding $\mathrm{C}$ peptide and $\mathrm{A}$ chain and green fluorescent protein gene (GFP) (exogenous gene control) fragment were cloned into pGEM-T Easy Vector (Promega). dsRNA synthesis was performed using T7 RNA Polymerase (Takara) and SP6 RNA Polymerase (TaKaRa) following the standard protocols. Finally, dsRNA was diluted with $10 \mathrm{mM}$ phosphate-buffered saline (PBS, pH 7.4).

\section{Short-Term Silencing Experiment in vivo}

The efficacy of gene knockdown via RNAi was evaluated by a short-term silencing experiment carried out with $L$. vittata at gonadal development stage I. A total of 15 shrimp (carapace 
length: $3.21 \pm 0.35 \mathrm{~mm}$, body weight: $50.40 \pm 12.14 \mathrm{mg}$ ) were randomly and equally assigned to evaluate the efficacy of gene knockdown via RNAi, we prepared a short-term silencing experiment using L. vittata at gonadal development stage I. A total of 15 shrimp (carapace length: $3.21 \pm 0.35 \mathrm{~mm}$, bodyweight: $50.40 \pm 12.14 \mathrm{mg}$ ) were randomly and equally assigned to the following 3 treatment groups $(n=5)$ : dsRNA Lvit-IAG2-injected, dsRNA GFP-injected, and PBS. The delivery of dsRNA (2 $\mu \mathrm{g} / \mathrm{g}$ ) (Liu et al., 2020) was by intramuscular injection in the abdominal segment of shrimp, and the PBS-injected treatment received an equivalent volume of PBS. Sampling was performed $24 \mathrm{~h}$ after injection. AG and eyestalk ganglion were collected after the shrimp were anesthetized on ice for $5 \mathrm{~min}$. Expression levels of LvitIAG1 (GenBank accession number: MT114196) and Lvit-IAG2 in the AG were detected by qRT-PCR to test the efficacy and specificity of dsRNA-mediated silencing on Lvit-IAG2. Meanwhile, Lvit-GIH1 (GenBank accession number: MT113121), Lvit-GIH2 (GenBank accession number: MT313290), Lvit-CHH (GenBank accession number: MT701562), Lvit-CFSH1 (GenBank accession number: MT114198) and Lvit-CFSH2 (GenBank accession number: MT114199) expression levels in the eyestalk ganglion were also detected. RNA extraction, qRT-PCR, and the first-strand cDNA were performed as described in section "Expression Profile of Lvit-IAG2 and Lvit-IAG1 During Gonadal Development.”

\section{Long-Term Silencing Experiment in vivo}

A long-term silencing experiment was prepared to explore the potential role of Lvit-IAG2 in sexual differentiation and gonadal development in L. vittata. Shrimp (carapace length $3.07 \pm 0.20 \mathrm{~mm}$, bodyweight $45.98 \pm 7.43 \mathrm{mg}$ ) at stage I were randomly categorized into 3 treatment groups $(n=11)$ as described in section "Short-Term Silencing Experiment in vivo." An equivalent dose of dsRNA $(2 \mu \mathrm{g} / \mathrm{g})$ or equivoluminal PBS was injected into the abdominal segment of shrimp once every 4 days (for a total of 8 injections in a 29 day duration); during which shrimp were kept in seawater aquaria under the following conditions: Temperature, $25.5 \pm 0.5^{\circ} \mathrm{C}$; salinity, $32 \pm 1$ PSU; a 12L:12D photoperiod. The shrimp were fed with a commercially formulated shrimp diet twice a day. On day 30 (24 $\mathrm{h}$ after the 8 th injection), all shrimps were sampled after anesthetization. Measurements of carapace length and body weight were recorded. Male and female external sexual characteristics and gonad shape were photographed using a stereomicroscope (model M165FC; Leica Application Suite X). Hematoxylin-eosin ( $\mathrm{H} \& \mathrm{E})$ staining was applied to visualize morphological and histological changes in ovotestis. The long and short axis lengths of each vitellogenic oocyte were measured and averaged, yielding a mean diameter for each cell. Only cells with visible nucleus were measured. The vitellogenic oocyte diameter of each individual was then calculated from 5 cells/field and 3 fields/section. Samples of AG, eyestalk ganglion, the ovarian region of the gonad, and hepatopancreas were collected to examine the relative mRNA expression levels of Lvit-IAG2, Lvit-GIH1, Lvit-GIH2, Lvit-CHH, Lvit-CFSH1, LvitCFSH2, Lvit-Vg (GenBank accession number: MT113122), and
Lvit-VgR (GenBank accession number: MT114195) by qRTPCR. RNA extraction, the first-strand cDNA synthesis, and qRT-PCR analyses were performed as described in section "Expression Profile of Lvit-IAG2 and Lvit-IAG1 During Gonadal Development.”

\section{Statistical Analyses}

Normality of data was established by the Kolmogorov-Smirnov test. All the data were presented in a normal distribution and tested for variances homogeneity by the Levene's test. All statistical analyses were performed using the SPSS 18.0 software; statistical significance $(p<0.05)$ of the data was determined using one-way ANOVA followed by Tukey's multiple range tests. All data were presented as mean $\pm \operatorname{SEM}(n=4-6)$.

\section{RESULTS}

\section{Sequence Analysis of Lvit-IAG2}

A schematic diagram of preproprotein of Lvit-IAG2 was depicted in Figure 1A. The Lvit-IAG2 (GenBank accession number: MT114197) coding region was 441-bp in length and encoded a 146-aa polypeptide, including a 27 -aa signal peptide, 36-aa B chain, 45-aa C peptide, and 38-aa A chain. We predicted 6 conserved cysteine residues in the $\mathrm{B}$ chain $\left(\mathrm{C}_{\mathrm{B} 12}\right.$ and $\left.\mathrm{C}_{\mathrm{B} 23}\right)$ and A chain $\left(\mathrm{C}_{\mathrm{A} 13}, \mathrm{C}_{\mathrm{A} 14}, \mathrm{C}_{\mathrm{A} 19}\right.$, and $\left.\mathrm{C}_{\mathrm{A} 27}\right)$. The predicted mature Lvit-IAG2 peptide comprised the $\mathrm{B}$ and $\mathrm{A}$ chains with two interchain disulfide bonds (between $\mathrm{C}_{\mathrm{B} 12}$ and $\mathrm{C}_{\mathrm{A} 14}, \mathrm{C}_{\mathrm{B} 23}$, and $\mathrm{C}_{\mathrm{A} 27}$, respectively) and an intrachain disulfide bond (between $\mathrm{C}_{\mathrm{A} 13}$ and $\left.\mathrm{C}_{\mathrm{A} 19}\right)$.

\section{Homology and Phylogenetic Analysis}

Multiple sequence alignment of the putative B chain and A chain of IAGs from decapod species was highlighted in Figure 1B. Notably, 6 cysteine residues forming two interchain disulfide bonds (between $\mathrm{C}_{\mathrm{B} 15}$ and $\mathrm{C}_{\mathrm{A} 18}, \mathrm{C}_{\mathrm{B} 26}$ and $\mathrm{C}_{\mathrm{A} 37}$, respectively) and an intrachain disulfide bond (between $\mathrm{C}_{\mathrm{A} 18}$ and $\mathrm{C}_{\mathrm{A} 28}$ ) were fully conserved among decapod species. Besides, Lvit-IAG2 shared the highest identity with Lvit-IAG1 (58.82\% for B chain and $44.12 \%$ for A chain, respectively). Based on phylogenetic analysis, insulin and related peptides formed four major clades: IAG, insulin, gonadulin, and relaxin. Meanwhile, the IAGs in decapods formed three subclades: (i) The family Caridea; (ii) sequences from the family Brachyura; (iii) the families Astacidea, Achelata, and Penaeoidea (Figure 2). We classified Lvit-IAG2 into the subclade containing the family Caridea.

\section{Spatial and Temporal Expression Profiles of Lvit-IAG2}

To explore the spatial distribution profiles of Lvit-IAG2, RT-PCR was performed on L. vittata at gonadal development stage I. Results demonstrated exclusive expression of Lvit-IAG2 in the AG (Figure 3A). The relative expression of Lvit-IAG2 in the AG during gonadal development was also assessed through qRTPCR (Figure 3B). Notably, the expression levels of Lvit-IAG2 


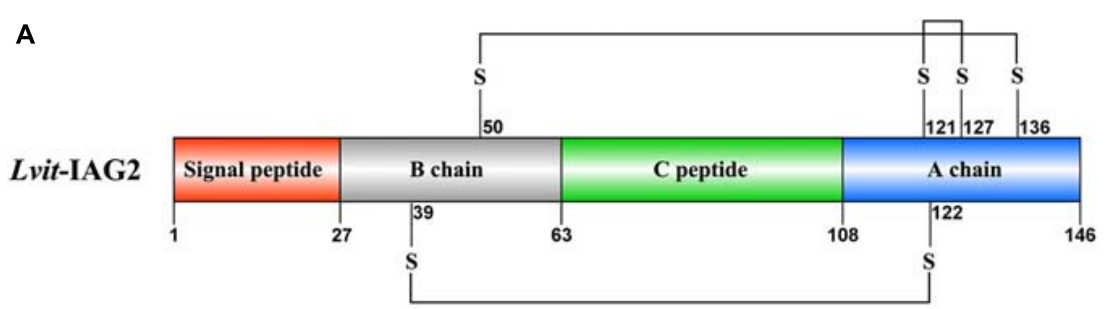

B

\section{B chain}

Es-IAG
Cqui-IAG
Sp-IAG
Cs-IAG
Sv-IAG
Je-IAG
Cd-IAG
Cqua-IAG
Pc-IAG
Pf-IAG
Ppac-IAG
Pp-IAG
Ml-IAG
Ppau-IAG
Mr-IAG
Mv-IAG
Lw-IAG
Mn-IAG
Pm-IAG
Pj-IAG
Pch-IAG1
Pch-IAG2
Pv-IAG
Lvir-IAG1
Lvit-IAG2

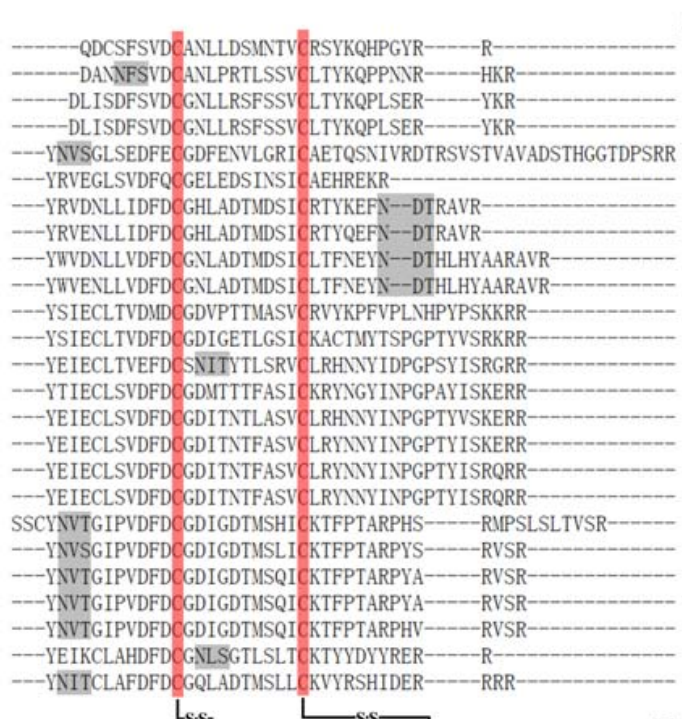

Identity to Lvit-IAG2

$35.48 \%$

$30.30 \%$

$32.35 \%$

$32.35 \%$

$27.78 \%$

33.33\%

$45.16 \%$

$45.16 \%$

$41.18 \%$

$41.18 \%$

$38.89 \%$

$38.89 \%$

$38.89 \%$

$41.67 \%$

$36.11 \%$

$38.89 \%$

$41.67 \%$

$41.67 \%$

$41.67 \%$

$41.67 \%$

$41.67 \%$

$41.67 \%$

$41.67 \%$

$58.82 \%$

A chain

Es-IAG

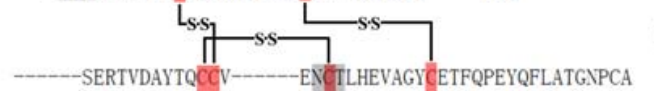

Identity to Lvit-IAG2

Cqui-IAG

Sp-IAG

Cs-IAG

SV-IAG

Je-IAG

Cd-IAG

Cqua-IAG

Pc-IAG

Pf-IAG

Ppac-IAG

Pp-IAG

MI-IAG

Ppau-IAG

Mr-IAG

Mv-IAG

Lw-IAG

Mn-IAG

Pm-IAG

Pj-IAG

Pch-IAGI

Pch-IAG2

Pv-IAG

Lvit-IAG1

Lvit-IAG2

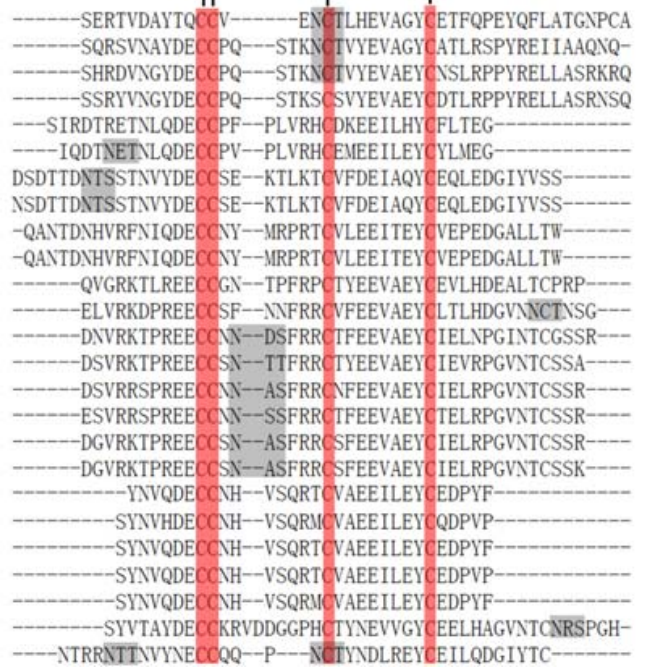

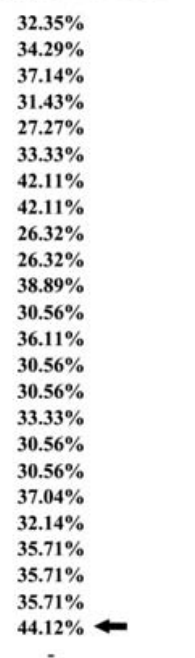

FIGURE 1 | Sequence analysis of Lvit-IAG2. (A) Schematic diagram of preproprotein of Lvit-IAG2 containing a signal peptide, B chain, C peptide, and A chain. The six cysteine residues of $B$ chain and $A$ chain were predicted to form three putative disulfide bridges connected with lines. (B) Multiple sequence alignment of putative B chain and A chain of decapod IAGs. The sequence information was given in Table 2. Six conserved cysteine residues were boxed in red, and three putative disulfide bridges were connected with lines. Predicted N-glycosylation sites were marked by gray shadow. Lvit-IAG1 was marked with solid arrows.

reached a peak at stage I, decreased sharply at stage II, and were continuously maintained at low levels at stages III and $\operatorname{IV}\left[F_{(3,16)}=19.797, p<0.05\right]$. Similar trend was observed in Lvit-IAG1 expression profiles except that the sharp decrease presented at stage III $\left[F_{(3,16)}=161.408, p<0.05\right]$, which was consistent with results of the former study (Liu et al., 2020). 


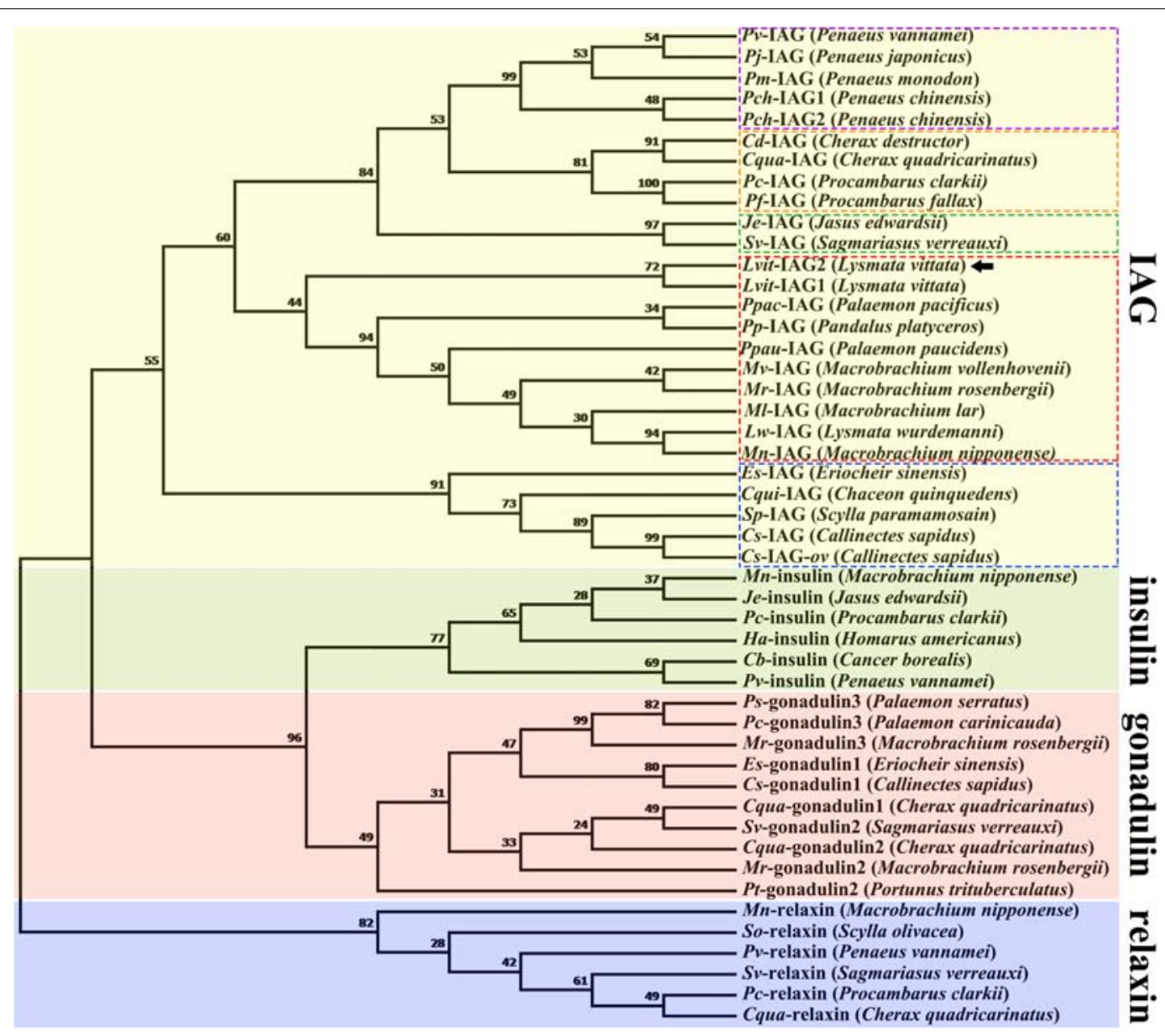

FIGURE 2 | Phylogenetic tree of amino acid sequences of the insulin and related peptide in decapods. Except for Lvit-IAG2 (GenBank accession number: MT114197, Lysmata vittata) and Cs-IAG-ov (GenBank accession number: APC42865.1, Callinectes sapidus), IAG sequences shown in Table 2; other sequences were obtained from Veenstra (2020). Deduced amino acid sequences of insulin and related peptide excluding signal peptide were used for phylogenic analysis. Phylogenetic analysis was conducted by Maximum Likelihood method based on the JTT matrix-based model in MEGA7. The percentage of replicate trees in which the associated taxa clustered together in the bootstrap test $(1,000$ replicates) was shown next to the branches. Four major clades were represented by different colors. Each subclade of IAGs was noted by a different color of dashed box. Lvit-IAG2 was marked by a solid arrow.

\section{Short-Term Silencing Experiment in vivo}

Results demonstrated that the transcript level of Lvit-IAG2 was explicitly inhibited up to $77 \%\left[F_{(2,12)}=22.851, p<0.05\right]$. Meanwhile, Lvit-IAG2 knockdown significantly suppressed the expression of Lvit-CHH $\left[F_{(2,12)}=113.857, p<0.05\right]$. On the contrary, no significant difference in Lvit-IAG1 $\left[F_{(2,12)}=0.173\right.$, $p>0.05]$, Lvit-CFSH1 $\left[F_{(2,12)}=0.876, p>0.05\right]$, Lvit-CFSH2 $\left[F_{(2,12)}=2.270, p>0.05\right]$, Lvit-GIH1 $\left[F_{(2,12)}=0.687, p>0.05\right]$, and Lvit-GIH2 $\left[F_{(2,12)}=1.378, p>0.05\right]$ expression was found (Figure 4).

\section{Long-Term Silencing Experiment in vivo Effects of Lvit-IAG2 Silencing on Gene Expression}

At the end of the 30 day long-term trial, the efficacy of gene knockdown was evaluated. Compared to the PBS treatment, Lvit-IAG2 transcript was $95 \%$ inhibited $\left[F_{(2,}, 13\right)=172.195$, $p<0.05]$. Besides, Lvit-IAG2 knockdown not only significantly inhibited the expression of Lvit-CHH in the eyestalk ganglion
$\left[F_{(2,13)}=38.197, p<0.05\right]$ but also repressed $L v i t-V g$ expression in the hepatopancreas $\left[F_{(2,13)}=6.851, p<0.05\right]$ and Lvit-VgR expression in the ovarian region $\left[F_{(2,13)}=9.605, p<0.05\right]$. However, we found no significant difference in the expression levels of Lvit-CFSH1 $\left[F_{(2,13)}=0.953, p>0.05\right], \operatorname{Lvit-CFSH} 2\left[F_{(2}\right.$, 13) $=1.129, p>0.05]$, Lvit-GIH1 $\left[F_{(2,13)}=1.333, p>0.05\right]$, and Lvit-GIH2 $\left[F_{(2,13)}=1.249, p>0.05\right]$; similar results were reported with short-term silencing experiment (Figures 4, 5).

\section{Effects of Lvit-IAG2 Silencing on Growth and Development of Sexual Characteristics}

After a 30 day long-term experiment, we recorded the average carapace length and bodyweight of the shrimps. Shrimps from the Lvit-IAG2 silencing treatment group $(4.27 \pm 0.05 \mathrm{~mm}$, $90.05 \pm 3.08 \mathrm{mg}$ ) were much smaller compared to those of the PBS $(4.83 \pm 0.05 \mathrm{~mm}, 144.78 \pm 3.04 \mathrm{mg})$ or dsRNA GFP $(4.96 \pm 0.05 \mathrm{~mm}, 147.87 \pm 4.33 \mathrm{mg})$ treatment groups [carapace length: $F_{(2,13)}=7.619, p<0.05$; body weight: 

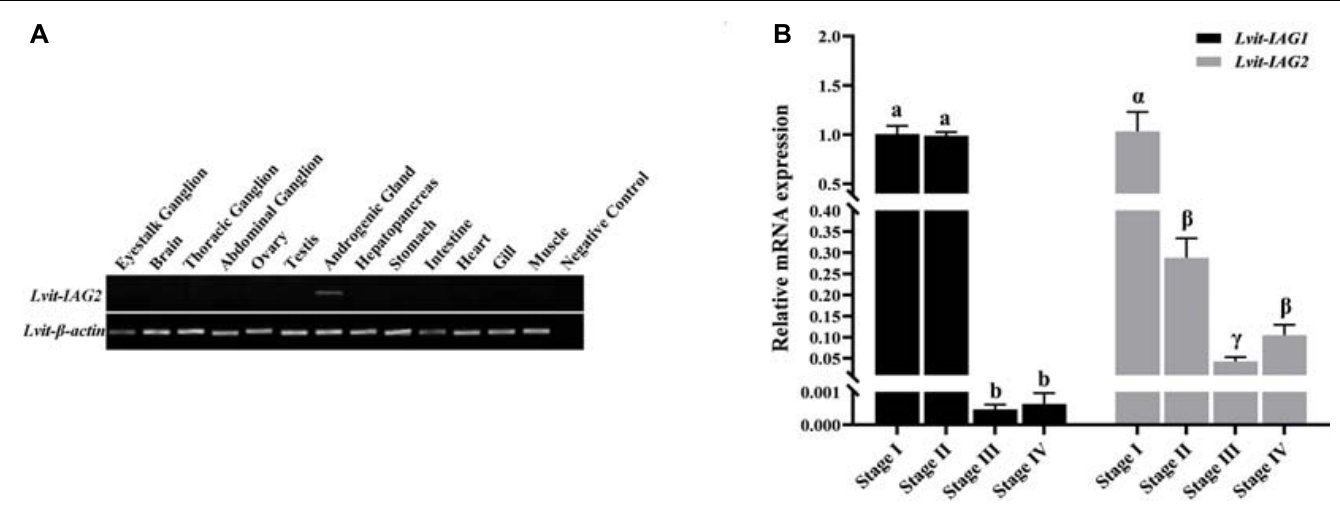

FIGURE 3 | Spatial expression profile of $L$ vit-IAG2 and temporal expression profiles of Lvit-IAG2 and Lvit-IAG1. (A) Distribution of $L$ vit-IAG2 in different tissues of $L$. vittata. The analysis was generated by PCR assays with cDNAs from various tissues of individuals at the gonadal development stage I. The $L v i t-\beta$-actin gene was used as a reference control gene. (B) Expression profiles of $L$ vit-IAG2 and Lvit-IAG1 in the androgenic gland during gonad development by qRT-PCR. The $L$ vit-IAGs expression levels standardized by Lvit- $\beta$-actin expression levels were represented as mean \pm SEM ("a, b, and c," $p<0.05$; " $\alpha, \beta$, and $\gamma$," $p<0.05$; one-way ANOVA followed by Tukey's multiple range tests; $n=5)$.

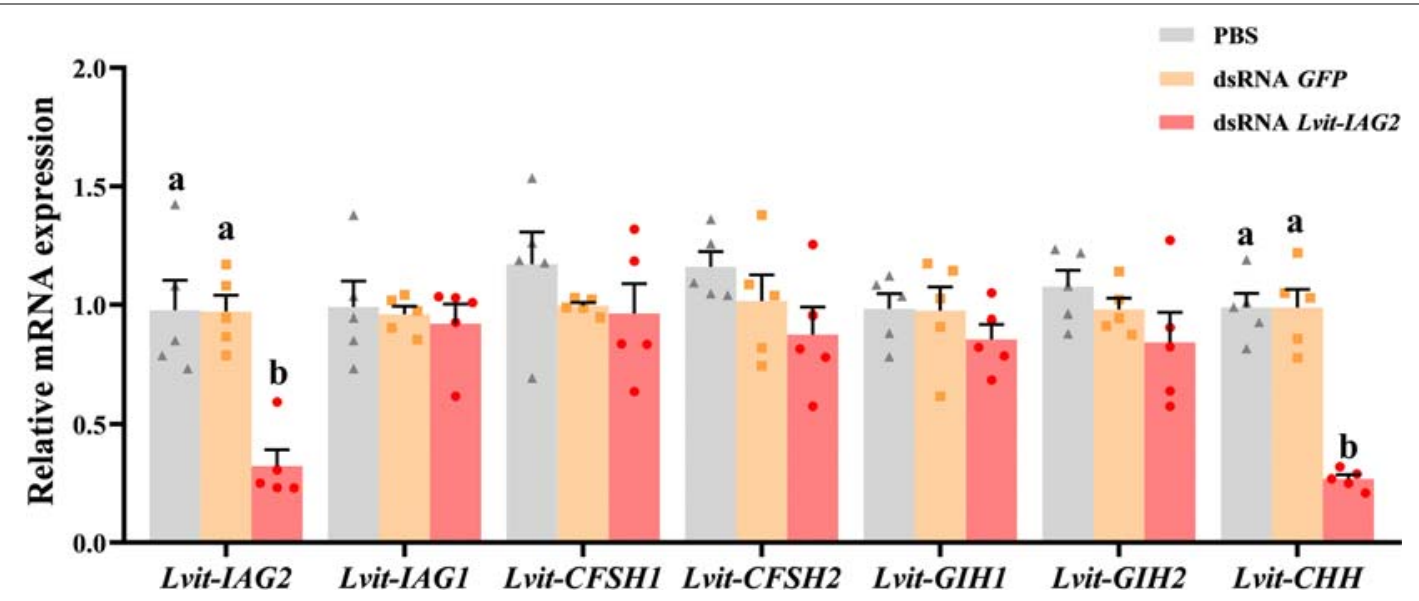

FIGURE 4 | Effects of short-term Lvit-IAG2 silencing on gene expression of $L$. vittata. The effectiveness of gene knockdown in the short-term $L$ vit-IAG2 silencing experiment was evaluated by qRT-PCR. The expression levels of Lvit-IAG2, Lvit-IAG1, Lvit-CFSH1, Lvit-CFSH2, Lvit-GIH1, Lvit-GIH2, and Lvit-CHH were detected following in vivo injection with PBS, dsRNA GFP or dsRNA Lvit-IAG2. The gene expression levels were standardized by $L$ vit- $\beta$-actin expression levels and represented as mean \pm SEM ("a and b," $p<0.05$; one-way ANOVA followed by Tukey's multiple range tests; $n=5$ ).

$\left.F_{(2,13)}=11.082, p<0.05\right]$ (Figures 6A,B). Moreover, changes in male and female sexual characteristics were documented at the end of the experiment through photography (Figure 7). Notably, Lvit-IAG2 gene knockdown retarded appendices masculinae (AM) development $\left[F_{(2,13)}=24.824, p<0.05\right]$ (Figures 6C, 7 ). No significant difference in female characteristics (female gonophores) and other male sexual characteristics (male gonophores and cincinnuli) was observed (Figure 7).

\section{Effects of Lvit-IAG2 Silencing on Gonadal Development}

We examined the morphological and histological characteristics of gonads following Lvit-IAG2 knockdown; notably, gene silencing resulted in abnormal development of gonad (Figure 8). In the testicular regions, different compositions of germ cell types were reported across the three treatments. For both
GFP-injected and PBS-injected control, active spermatogenesis occurred (evident from the abundant spermatogonia, spermatid, and spermatozoa found in the testicular region) (Figures 8D,E). However, with Lvit-IAG2 silencing, a large number of secondary spermatocytes and little spermatid were found, demonstrating arrested spermatogenesis (Figure 8F). Besides, the ovarian region was much smaller (Figure 8C), and in terms of the arrangement, germ cells were tighter and more disordered compared to those of the controls (Figure 8I). Additionally, the oocyte size significantly decreased following Lvit-IAG2 knockdown $\left[F_{(2}\right.$, 13) $=67.576, p<0.05]$ (Figures 6D, 8I).

\section{DISCUSSION}

Following a previous transcriptome study, two ILP transcripts, Lvit-IAG1 and Lvit-IAG2, exist in the PSH shrimp, L. vittata 


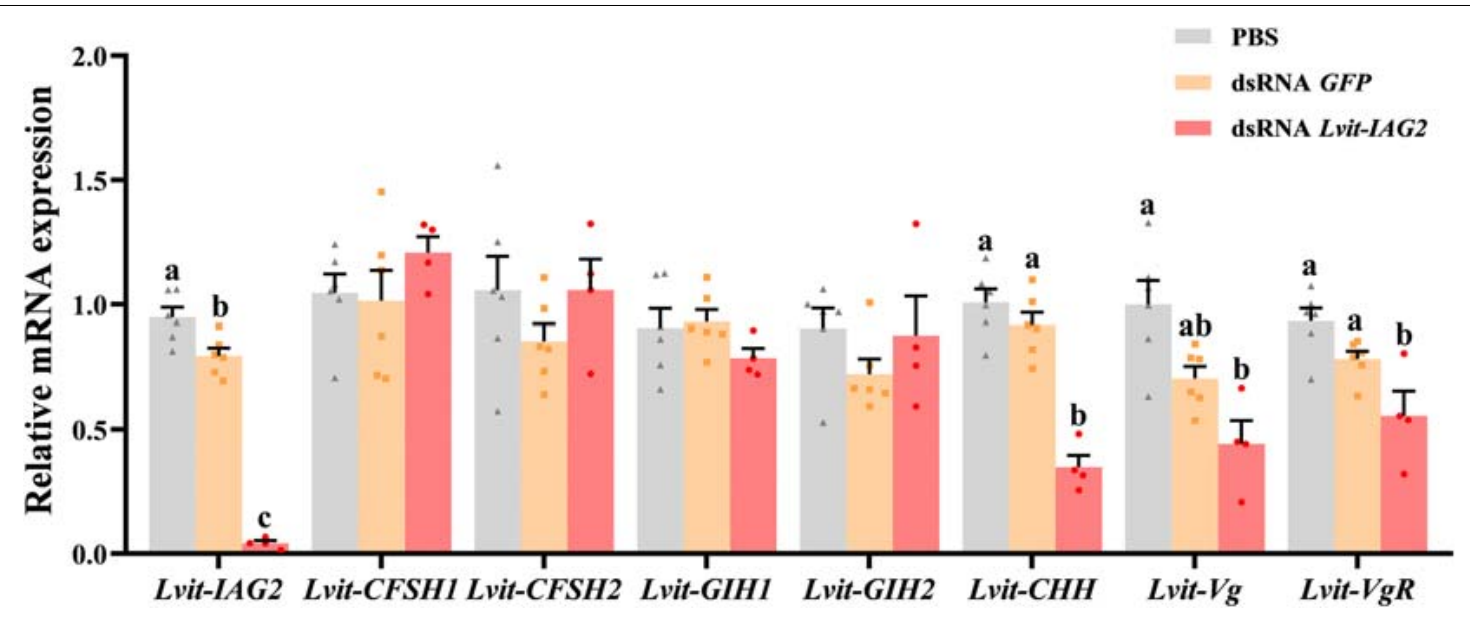

FIGURE 5 | Effects of long-term Lvit-IAG2 silencing on gene expression of $L$. vittata. The effectiveness of gene knockdown in the long-term $L$ vit-IAG2 silencing experiment was evaluated by qRT-PCR. The expression levels of Lvit-CFSH1, Lvit-CFSH2, Lvit-GIH1, Lvit-GIH2, Lvit-CHH, Lvit-Vg, and Lvit-VgR were detected following in vivo injection with PBS, dsRNA GFP or dsRNA Lvit-IAG2. The gene expression levels were standardized by $L$ vit- $\beta$-actin expression levels and represented as mean \pm SEM (“a, b, and c," $p<0.05$; one-way ANOVA followed by Tukey's multiple range tests; $n=4-6$ ).
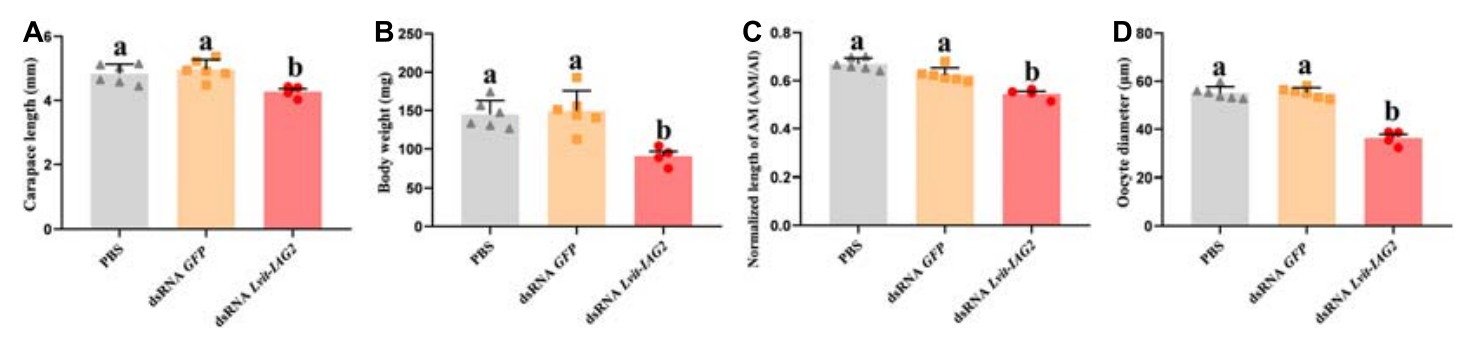

FIGURE 6 | Effects of Lvit-IAG2 silencing on carapace length, body weight, AM length and oocyte diameter of L. vittata. (A) Carapace length; (B) body weight; (C) the normalized length of AM (AM/Al); (D) the oocyte diameter. Data were represented as mean \pm SEM ("a and b," $p<0.05$; one-way ANOVA followed by Tukey's multiple range tests; $n=4-6$ ).

(Bao et al., 2020). Lvit-IAG1 has also been identified as an IAG via functional study (Liu et al., 2020). In this study, we expounded on the biological functions of another ILP, Lvit-IAG2. Lvit-IAG2 contained features that were structurally conserved in IAGs: a preproprotein comprised of a signal peptide, $\mathrm{B}$ chain, $\mathrm{C}$ peptide, and A chain; six strictly conserved cysteine residues forming three disulfide bonds in the $\mathrm{B}$ chain and A chain among predicted mature peptides. Besides, based on phylogenetic tree analysis, Lvit-IAG2 was classified into the IAG clade and subclade of the family Caridea. These results confirmed that Lvit-IAG2 was identified structurally as an IAG. Meanwhile, we noticed that some studies reported more than one transcript of IAG in a decapod species ( $\mathrm{Li}$ et al., 2012; Huang et al., 2017b). However, these transcripts were generated by alternative splicing of one IAG gene, and deduced mature amino acid sequences of these encoded IAG proteins were nearly identical (Li et al., 2012; Huang et al., 2017b). Herein, mature peptides of Lvit-IAG2 and LvitIAG1 shared only $58.82 \%$ similarity for B chain and $44.12 \%$ similarity for A chain, respectively. These results might suggest that two different IAG genes exist in the species, which has never been reported.
Earlier experiments demonstrated that $I A G$ genes were mainly expressed in the AG in decapod crustaceans (Manor et al., 2007). More recently, studies detected IAG mRNA expression in the hepatopancreas and ovary (Chung, 2014; Huang et al., 2014). Herein, the Lvit-IAG2 gene was exclusively expressed in the AG; its expression level was significantly higher in the male phase (Stage I-III) than that in the euhermaphrodite phase (Stage IV) during the life cycle of the PSH species. These reports implied that Lvit-IAG2 potentially can initiate male sexual differentiation in the male phase and maintain male reproductive activity in the euhermaphrodite phase. Notably, Lvit-IAG2 and Lvit-IAG1 displayed slightly different expression trends during gonadal development. The expression levels of Lvit-IAG2 decreased sharply at stage II, while significant downregulation of LvitIAG1 expression appeared at stage III. The distinction suggested different functions of the two IAGs.

The present results demonstrated that gene silencing via RNAi successfully induced a specific knockdown of Lvit-IAG2 transcripts levels by 77 and $95 \%$ in short-term and long-term silencing experiments, respectively. Furthermore, we assessed the influence of Lvit-IAG2 on the development of male features by comparing male external features and germ cell type composition 


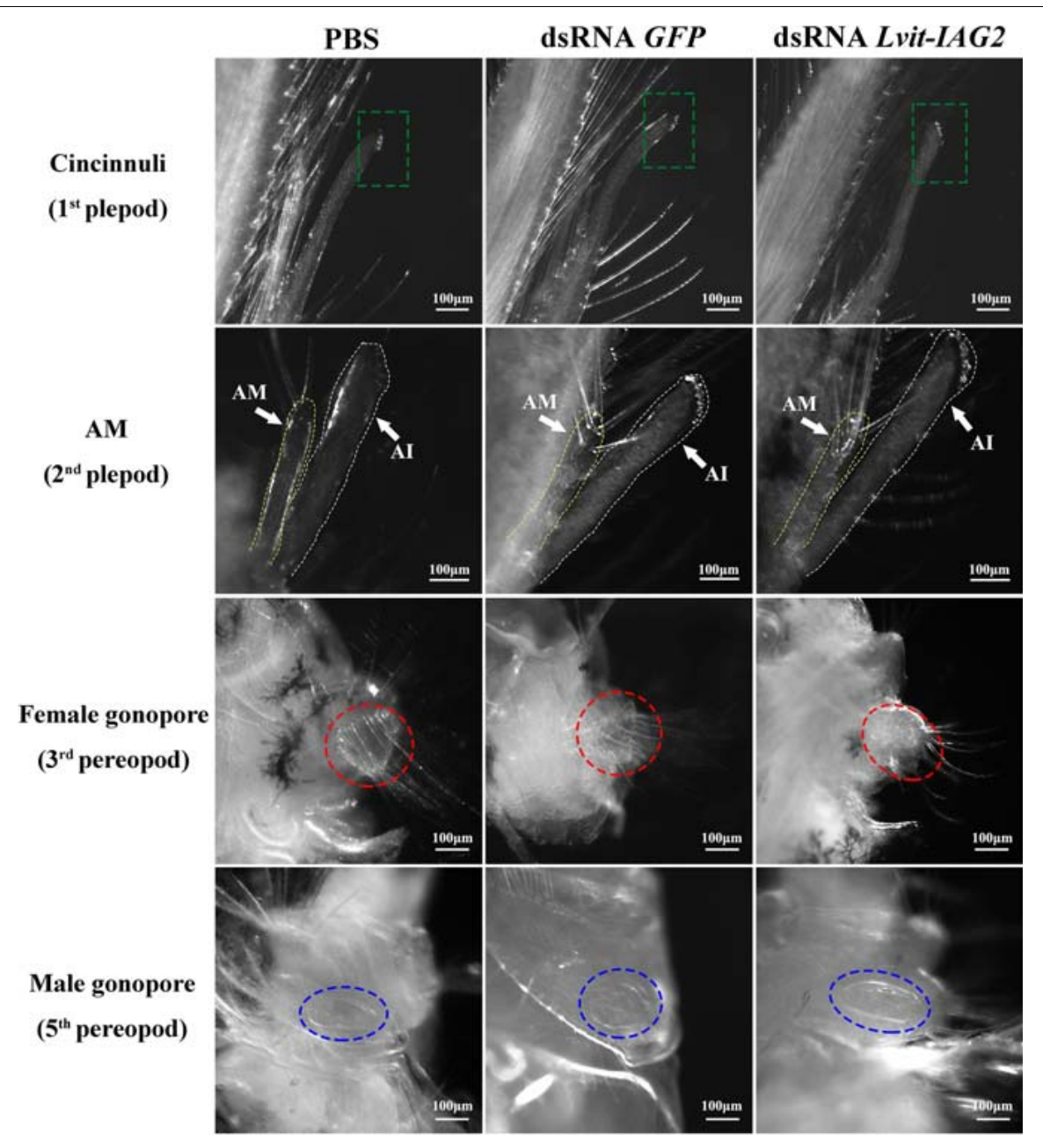

FIGURE 7 | Effects of Lvit-IAG2 silencing on development of male and female sexual characteristics. Both male (cincinnuli, AM, male gonopore) and female characteristics (female gonopore) were photographed at the end of the 30 day experiment. The Al was marked by white dashed lines, while the AM was marked by yellow dashed lines. Male and female gonopores were noted by blue and red dotted circle, respectively. AM, appendices masculinae; Al, appendix interna.

in the testicular region. Notably, previous studies revealed that IAG silencing delayed the appearance of male sexual characteristics and induced testicular spermatogenesis arrest in the freshwater prawn, M. rosenbergii (Ventura et al., 2009) and the red swamp crayfish Procambarus clarkii (Shi et al., 2019). Similar results were observed for Lvit-IAG1 in L. vittata. Lvit-IAG1 knockdown induced abnormal development of both $\mathrm{AM}$ and male gonopores, and suppressed germ cells at the primary spermatocytes (Liu et al., 2020). However, we found that Lvit-IAG2 and Lvit-IAG1 exerted distinct functions in the male sexual differentiation process. Lvit-IAG2 knockdown solely suppressed AM development and germ cells arrested at the secondary spermatocytes, implying its crucial contribution to secondary spermatocyte-to-spermatid transition. These results demonstrated that Lvit-IAG2 was indeed another functional IAG of L.vittata. On the other hand, either primary-to-secondary spermatocyte transition or secondary spermatocyte-to-spermatid transition was essential for the normal development of testicular region. Meanwhile, both of the two Lvit-IAGs might be involved in developmental processes specific to AM. Thus, Lvit-IAG2 and Lvit-IAG1 acted in concert, and both of them were indispensable in the male sexual differentiation process of the PSH species.

Simultaneously, we evaluated the effects of Lvit-IAG2 on female development; notably, no significant difference in LvitCFSHs gene expression among the three treatments in both short-term and long-term silencing experiments was reported. Further morphological characterization revealed similar results as Lvit-IAG2 knockdown did not impact the development of female gonopores. Nonetheless, a significant inhibitory effect on the development of ovarian region was observed. Contrary to the controls, shrimp in the Lvit-IAG2 silencing treatments exhibited decreased volume of the ovarian region, and germ cells were much smaller and arranged in a disorganized mass. It is interesting to note that silencing IAG gene in another hermaphrodite shrimp $P$. platyceros induced feminization of individuals, including $V g$ expression in the hepatopancreas and ovarian development (Levy et al., 2020a), which is consistent with studies in dioecious species. Different outcome might be 


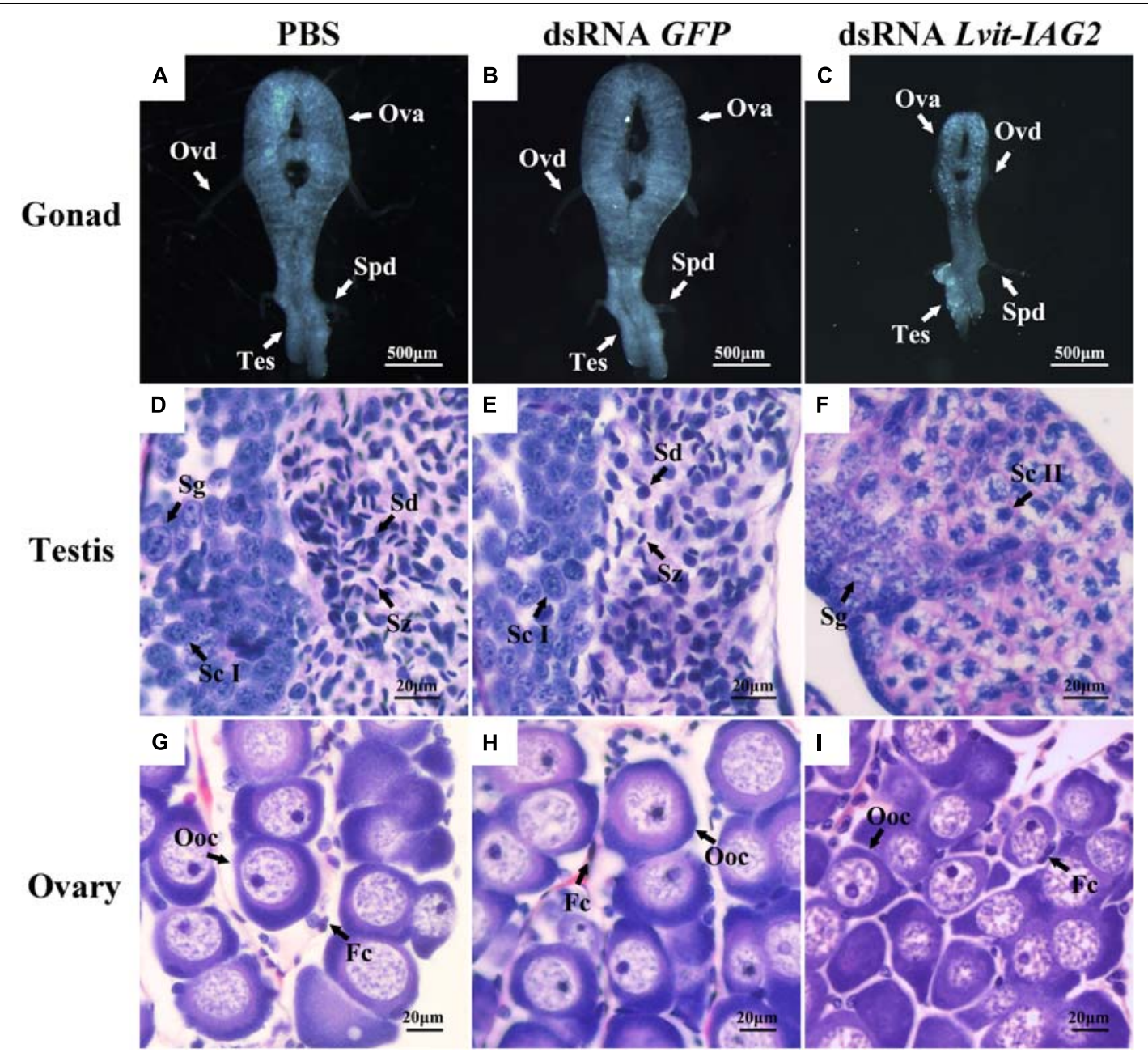

FIGURE 8 | Effects of Lvit-IAG2 silencing on gonadal development of $L$. vittata. Gonad morphology and characteristics were photographed at the end of the 30 day experiment following in vivo injection of PBS (A), dsRNA GFP (B), or dsRNA Lvit-IAG2 (C). Hematoxylin and eosin (H \& E)-stained sections was used for structure description. Testicular region mainly consisted of Sg and Sd/Sz in PBS (D) and dsRNA GFP (E) injection treatments while the majority of cells in the testicular region of dsRNA Lvit-IAG2 injected treatment were Sc II (F). Ovarian regions of the shrimp from the Lvit-IAG2 silencing treatment were more transparent and smaller in relative size (C). Compared with the PBS-injected (G) and dsRNA GFP-injected (H) group, oocytes were less developed and arranged more tightly and disorderly in the dsRNA Lvit-IAG2-injected group (I). Ova, ovary; Tes, testis; Ovd, oviduct; Spd, sperm duct; Oog, oogonia; Ooc, oocytes; Fc, follicular cell; Sg, spermatogonia; Sc I, primary spermatocyte; Sc II, secondary spermatocyte; Sd, spermatid; Sz, spermatozoa.

induced by the difference between SPH and PSH. For SPH shirimp $P$. platyceros, individuals first mature as male and eventually acquire reproductive function of female, indicating that functional males and females have different reproductive systems (Levy et al., 2020b). This phase-specific gender pattern is similar, to some extent, with dioecious species, and so it is with reproductive endocrine system. However, For SPH shrimp L. vitata, individuals finally acquire the same euhermaphrodite reproductive system with both male and female functions. It is therefore reasonable to speculate that different reproductive endocrine system presents in PSH species, and IAG is likely to possess different functions in ovarian development. Following reports from previous experiments, Lvit-IAG1 could regulate ovarian development by inhibiting Lvit-GIHs expression (Liu et al., 2020). However, in the present experiment, we reported no significant change in Lvit-GIHs transcript levels. Remarkably, knockdown of Lvit-IAG1 and Lvit-IAG2 suppressed ovarian development. Thus, we could not eliminate the possibility that insulin and related peptides could directly regulate $V g$ expression in the hepatopancreas, and promote oocyte growth and development. Following a review by Das and Arur (2017), insulin signaling played a pivotal role in the regulation of oocyte growth, development, and maturation in both vertebrates and invertebrates. For instance, in the rainbow trout Oncorhynchus mykiss, insulin was reported to induce the accumulation of vitellogenin in cultured oocytes (Shibata et al., 1993). Besides, ILPs could also control germline cyst growth and vitellogenesis in Drosophila (LaFever and Drummond-Barbosa, 2005). In the silkmoth Bombyx mori, knock-out of an insulin-like growth factor (IGF)-like peptide gene induced drastically lower ovary weights and number of eggs in female individuals (Fujinaga et al., 2019). In the kuruma shrimp Marsupenaeus japonicas, Mj-IAG 
was proved to have insulin-type disulfide, rather than $\mathrm{AGH}$ type (Katayama et al., 2014), indicating that IAG potentially possessed similar functions as IGF or insulin. Moreover, a recent in vitro experiment revealed that bovine insulin induced the $V g$ expression in the hepatopancreas of the mud crab S. paramamosain, implying that insulin-like peptide could directly modulate vitellogenesis in the hepatopancreas (Huang et al., 2017a). Thus, Lvit-IAG2 potentially possessed similar functions as IGF or insulin in the PSH L. vittata, which might be explained by their structural similarities (Martin et al., 1998, 1999; Manor et al., 2007). On the other hand, growth and reproduction are closely related (Michalakis et al., 2013). Herein, we noted that Lvit-IAG2 silencing induced significantly slower growth in the treated shrimp, implying that Lvit-IAG2 is potentially a growth regulator. Similar results have been reported in the freshwater prawn $M$. rosenbergii (Ventura et al., 2009) and the mud crab S. paramamosain (Huang et al., 2014), and with the function of insulin and related peptide in mammals (Bruning et al., 2000; Neirijnck et al., 2019), fishes (Reinecke, 2010), and insects (Wu and Brown, 2006). Moreover, transcript levels of Lvit-CHH significantly decreased following knock down of Lvit-IAG2 in both short-term and long-term experiment. $\mathrm{CHH}$ is defined as a metabolism associated hormone as its participation in multiple metabolic processes, including glycometabolism (Webster et al., 2012), lipid metabolism (Montiel-Arzate et al., 2020) and ammonia metabolism (Zhang et al., 2020). Of note, the low expression of $\mathrm{Lvit}$ - $\mathrm{CHH}$ implied the growth and developmental abnormalities of individuals. Hence, it could also be reasonably hypothesized that retardation in the development of the ovarian region in Lvit-IAG2 silencing shrimp potentially arose from the abnormity in the growth and development of the shrimp. Further in vitro experiments are required to test the two hypotheses.

Taken together, the present work characterized an IAG gene, Lvit-IAG2, from the AG of the peppermint shrimp L. vittata and explored its crucial functions in regulating primary and secondary sexual characteristics of the PSH species. Moreover, this study implicated Lvit-IAG2 and LvitIAG1 to act in concert, aimed at regulating male sexual differentiation in the PSH shrimp. Also, Lvit-IAG2 may directly

\section{REFERENCES}

Alves, D. F. R., López Greco, L. S., Barros-Alves, S., de, P., and Hirose, G. L. (2019). Sexual system, reproductive cycle and embryonic development of the red-striped shrimp Lysmata vittata, an invader in the western Atlantic Ocean. PLoS One 14:e0210723. doi: 10.1371/journal.pone.0210723

Bao, C., Liu, F., Yang, Y., Lin, Q., and Ye, H. (2020). Identification of peptides and pheir GPCRs in the peppermint shrimp Lysmata vittata, a protandric simultaneous hermaphrodite species. Front. Endocrinol. 11:229. doi: 10.3389/ fendo.2020.00226

Bauer, R. T. (2000). Simultaneous hermaphroditism in Caridean shrimps: a unique and puzzling sexual system in the Decapoda. J. Crustac. Biol. 20, 116-128. doi: 10.1163/1937240X-90000014

Bruning, J. C., Gautam, D., Burks, D. J., Gillette, J., Schubert, M., Orban, P. C., et al. (2000). Role of brain insulin receptor in control of body weight and reproduction. Science 289, 2122-2125. doi: 10.1126/science.289.5487.2122

Chen, D., Liu, F., Zhu, Z., Lin, Q., Zeng, C., and Ye, H. (2019). Ontogenetic development of gonads and external sexual characters of the protandric simultaneous hermaphrodite peppermint shrimp, Lysmata vittata (Caridea: Hippolytidae). PLoS One 14:e0215406. doi: 10.1371/journal.pone.0215406 contribute to ovarian development, or via the promotion of growth in $L$. vittata. These findings provide new insights into molecular mechanisms of sexual differentiation in PSH crustaceans, expounding our current knowledge on crustacean reproductive endocrinology.

\section{DATA AVAILABILITY STATEMENT}

The datasets presented in this study can be found in online repositories. The names of the repository/repositories and accession number(s) can be found in the article/supplementary material.

\section{AUTHOR CONTRIBUTIONS}

FL contributed to conceptualization, methodology, software, validation, formal analysis, investigation, data curation, visualization and writing - original draft preparation of the study. HY contributed to conceptualization, methodology, validation, data curation, writing-review and editing, supervision, project administration and funding acquisition. WS contributed to investigation. AL contributed to validation and writing-review and editing of the study. ZZ contributed to funding acquisition and provided resources. All authors contributed to manuscript revision, read, and approved the submitted version.

\section{FUNDING}

The work was supported by the special fund of marine and fishery structure adjustment in Fujian (2020HYJG01 and 2020HYJG08).

\section{ACKNOWLEDGMENTS}

We also thank all laboratory members for their constructive suggestions and discussions.

Chung, J. S. (2014). An insulin-like growth factor found in hepatopancreas implicates carbohydrate metabolism of the blue crab Callinectes sapidus. Gen. Comp. Endocrinol. 199, 56-64. doi: 10.1016/j.ygcen.2014.01.012

Comeau, M., and Benhalima, K. (2018a). Functional anatomy of the female reproductive system of the American lobster (Homarus americanus). J. Morphol. 279, 1603-1614. doi: 10.1002/jmor.20889

Comeau, M., and Benhalima, K. (2018b). Functional anatomy of the male reproductive system of the American lobster (Homarus americanus). J. Morphol. 279, 1431-1443. doi: 10.1002/jmor.20878

Das, D., and Arur, S. (2017). Conserved insulin signaling in the regulation of oocyte growth, development, and maturation. Mol. Reprod. Dev. 84, 444-459. doi: $10.1002 / \mathrm{mrd} .22806$

Das, R., Krishna, G., Priyadarshi, H. P., Babu, G., Pavan-Kumar, A., Rajendran, K. V., et al. (2015). Captive maturation studies in Penaeus monodon by GIH silencing using constitutively expressed long hairpin RNA. Aquaculture 448, 512-520. doi: 10.1016/j.aquaculture.2015.06.036

Fu, C., Li, F., Wang, L., Wu, F., Wang, J., Fan, X., et al. (2020). Molecular characteristics and abundance of insulin-like androgenic gland hormone and effects of RNA interference in Eriocheir sinensis. Anim. Reprod. Sci. 215:106332. doi: 10.1016/j.anireprosci.2020.106332 
Fujinaga, D., Shiomi, K., Yagi, Y., Kataoka, H., and Mizoguchi, A. (2019). An insulin-like growth factor-like peptide promotes ovarian development in the silkmoth Bombyx mori. Sci. Rep. 9:18446. doi: 10.1038/s41598-019-54962-w

Guo, Q., Li, S., Lv, X., Xiang, J., Manor, R., Sagi, A., et al. (2019). Sex-biased CHHs and their putative receptor regulate the expression of IAG gene in the Shrimp Litopenaeus vannamei. Front. Physiol. 10:1525. doi: 10.3389/fphys.2019.01525

Huang, X., Feng, B., Huang, H., and Ye, H. (2017a). In vitro stimulation of vitellogenin expression by insulin in the mud crab, Scylla paramamosain, mediated through PI3K/Akt/TOR pathway. Gen. Comp. Endocrinol. 250, 175180. doi: 10.1016/j.ygcen.2017.06.013

Huang, X., Ye, H., and Chung, J. S. (2017b). The presence of an insulin-like androgenic gland factor (IAG) and insulin-like peptide binding protein (ILPBP) in the ovary of the blue crab, Callinectes sapidus and their roles in ovarian development. Gen. Comp. Endocrinol. 249, 64-70. doi: 10.1016/j.ygcen.2017.05. 001

Huang, X., Ye, H., Huang, H., Yang, Y., and Gong, J. (2014). An insulinlike androgenic gland hormone gene in the mud crab, Scylla paramamosain, extensively expressed and involved in the processes of growth and female reproduction. Gen. Comp. Endocrinol. 204, 229-238. doi: 10.1016/j.ygcen.2014. 06.002

Jiang, Q., Lu, B., Lin, D., Huang, H., Chen, X., and Ye, H. (2020). Role of crustacean female sex hormone (CFSH) in sex differentiation in early juvenile mud crabs, Scylla paramamosain. Gen. Comp. Endocrinol. 289:113383. doi: 10.1016/j.ygcen. 2019.113383

Juchault, P. (1999). Hermaphroditism and gonochorism. a new hypothesis on the evolution of sexuality in Crustacea. C. R. Acad. Sci. III 322, 423-427. doi: 10.1016/S0764-4469(99)80078-X

Katayama, H., Kubota, N., Hojo, H., Okada, A., Kotaka, S., Tsutsui, N., et al. (2014). Direct evidence for the function of crustacean insulin-like androgenic gland factor (IAG): total chemical synthesis of IAG. Bioorg. Med. Chem. 22, 5783-5789. doi: 10.1016/j.bmc.2014.09.031

Khalaila, I., Manor, R., Weil, S., Granot, Y., Keller, R., and Sagi, A. (2002). The eyestalk-androgenic gland-testis endocrine axis in the crayfish Cherax quadricarinatus. Gen. Comp. Endocrinol. 127, 147-156. doi: 10.1016/S00166480(02)00031-X

LaFever, L., and Drummond-Barbosa, D. (2005). Direct control of germline stem cell division and cyst growth by neural insulin in Drosophila. Science 309, 1071-1073. doi: 10.1126/science. 1111410

Levy, T., and Sagi, A. (2020). The "IAG-Switch"-a key controlling element in decapod crustacean sex differentiation. Front. Endocrinol. 11:651. doi: 10.3389/ fendo.2020.00651

Levy, T., Rosen, O., Simons, O., Savaya Alkalay, A., and Sagi, A. (2017). The gene encoding the insulin-like androgenic gland hormone in an all-female parthenogenetic crayfish. PLoS One 12:e0189982. doi: 10.1371/journal.pone. 0189982

Levy, T., Tamone, S. L., Manor, R., Aflalo, E. D., Sklarz, M. Y., Chalifa-Caspi, V., et al. (2020a). The IAG-switch and further transcriptomic insights into sexual differentiation of a protandric shrimp. Front. Mar. Sci. 7:587454. doi: 10.3389/fmars.2020.587454

Levy, T., Tamone, S. L., Manor, R., Bower, E. D., and Sagi, A. (2020b). The protandric life history of the Northern spot shrimp Pandalus platyceros: molecular insights and implications for fishery management. Sci. Rep. 10:1287. doi: 10.1038/s41598-020-58262-6

Li, F., Bai, H., Zhang, W., Fu, H., Jiang, F., Liang, G., et al. (2015). Cloning of genomic sequences of three crustacean hyperglycemic hormone superfamily genes and elucidation of their roles of regulating insulin-like androgenic gland hormone gene. Gene 561, 68-75. doi: 10.1016/j.gene.2015.02.012

Li, S., Li, F., Sun, Z., and Xiang, J. (2012). Two spliced variants of insulinlike androgenic gland hormone gene in the Chinese shrimp, Fenneropenaeus chinensis. Gen. Comp. Endocrinol. 117, 246-255. doi: 10.1016/j.ygcen.2012.04. 010

Liu, A., Liu, J., Liu, F., Huang, Y., Wang, G., and Ye, H. (2017). Crustacean female sex hormone from the mud crab Scylla paramamosain is highly expressed in prepubertal males and inhibits the development of androgenic gland. Front. Physiol. 9:924. doi: 10.3389/fphys.2018.00924

Liu, F., Shi, W., Ye, H., Zeng, C., and Zhu, Z. (2020). Insulin-like androgenic gland hormone 1 (IAG1) regulates sexual differentiation in a hermaphrodite shrimp through feedback to neuroendocrine factors. Gen. Comp. Endocrinol. 303:113706. doi: 10.1016/j.ygcen.2020.113706

Manor, R., Weil, S., Oren, S., Glazer, L., Aflalo, E. D., Ventura, T., et al. (2007) Insulin and gender: an insulin-like gene expressed exclusively in the androgenic gland of the male crayfish. Gen. Comp. Endocrinol. 150, 326-336. doi: 10.1016/ j.ygcen.2006.09.006

Martin, G., Sorokine, O., Moniatte, M., and Vandorsselaer, A. (1998). The androgenic hormone of the crustacean isopod Armadillidium vulgare. Ann. NY Acad. Sci. 839, 111-117. doi: 10.1111/j.1749-6632.1998.tb10741.x

Martin, G., Sorokine, O., Moniatte, M., Bulet, P., Hetru, C., and Van Dorsselaer, A. (1999). The structure of a glycosylated protein hormone responsible for sex determination in the isopod, Armadillidium vulgare. Eur. J. Biochem. 262, 727-736. doi: 10.1046/j.1432-1327.1999.00442.x

Michalakis, K., Mintziori, G., Kaprara, A., Tarlatzis, B. C., and Goulis, D. G. (2013). The complex interaction between obesity, metabolic syndrome and reproductive axis: a narrative review. Metabolism 62, 457-478. doi: 10.1016/j. metabol.2012.08.012

Montiel-Arzate, A., Sánchez-Castrejón, E., Camacho-Jiménez, L., Díaz, F., and Ponce-Rivas, E. (2020). Effect of recombinant crustacean hyperglycemic hormones $\mathrm{rCHH}-\mathrm{B} 1$ and $\mathrm{rCHH}-\mathrm{B} 2$ on lipid metabolism in the Pacific white shrimp Litopenaeus vannamei. Aquac. Res. 51, 4267-4278. doi: 10.1111/are. 14769

Nagaraju, G. P. C. (2011). Reproductive regulators in decapod crustaceans: an overview. J. Exp. Biol. 214, 3-16. doi: 10.1242/jeb.047183

Neirijnck, Y., Papaioannou, M. D., and Nef, S. (2019). The Insulin/IGF system in mammalian sexual development and reproduction. Int. J. Mol. Sci. 20:4440. doi: 10.3390/ijms20184440

Priyadarshi, H., Das, R., Pavan-Kumar, A., Gireesh-Babu, P., Javed, H., Kumar, S., et al. (2017). Silencing and augmentation of IAG hormone transcripts in adult Macrobrachium rosenbergii males affects morphotype transformation. J. Exp. Biol. 220, 4101-4108. doi: 10.1242/jeb.163410

Reinecke, M. (2010). Insulin-like growth factors and fish reproduction. Biol. Reprod. 82, 656-661. doi: 10.1095/biolreprod.109.08 0093

Rosen, O., Manor, R., Weil, S., Gafni, O., Linial, A., Aflalo, E. D., et al. (2010). A sexual shift induced by silencing of a single insulin-like gene in crayfish: ovarian upregulation and testicular degeneration. PLoS One 5:e15281. doi: 10. 1371/journal.pone.0015281

Shi, L., Han, S., Fei, J., Zhang, L., Ray, J. W., Wang, W., et al. (2019). Molecular characterization and functional study of insulin-like androgenic gland hormone gene in the red swamp crayfish, Procambarus clarkii. Genes 10:645. doi: 10.3390/ genes10090645

Shibata, N., Yoshikuni, M., and Nagahama, Y. (1993). Vitellogenin incorporation into oocytes of rainbow trout, Oncorhynchus mykiss, in vitro: effect of hormones on denuded oocytes. Dev. Growth Diff. 35, 115-121. doi: 10.1111/j.1440-169X. 1993.00115.x

Veenstra, J. A. (2020). Gonadulins, the fourth type of insulin-related peptides in decapods. Gen. Comp. Endocrinol. 296:113528. doi: 10.1016/j.ygcen.2020. 113528

Ventura, T., Manor, R., Aflalo, E. D., Weil, S., Raviv, S., Glazer, L., et al. (2009). Temporal silencing of an androgenic gland-specific insulin-like gene affecting phenotypical gender differences and spermatogenesis. Endocrinology 150, 1278-1286. doi: 10.1210/en.2008-0906

Ventura, T., Manor, R., Aflalo, E. D., Weil, S., Rosen, O., and Sagi, A. (2012). Timing sexual differentiation: full functional sex reversal achieved through silencing of a single insulin-like gene in the prawn, Macrobrachium rosenbergii1. Biol. Reprod. 86:90. doi: 10.1095/biolreprod.111.09 7261

Webster, S. G., Keller, R., and Dircksen, H. (2012). The CHH-superfamily of multifunctional peptide hormones controlling crustacean metabolism, osmoregulation, moulting, and reproduction. Gen. Comp. Endocrinol. 175, 217-233. doi: 10.1016/j.ygcen.2011.11.035

Wu, Q., and Brown, M. R. (2006). Signaling and function of insulin-like peptides in insects. Annu. Rev. Entomol. 51, 1-24. doi: 10.1146/annurev.ento.51.110104. 151011

Zhang, D., Sun, M., and Liu, X. (2017). Phase-specific expression of an insulinlike androgenic gland factor in a marine shrimp Lysmata wurdemanni: 
implication for maintaining protandric simultaneous hermaphroditism. PLoS One 12:e0172782. doi: 10.1371/journal.pone.0172782

Zhang, X., Pan, L., Wei, C., Tong, R., Li, Y., Ding, M., et al. (2020). Crustacean hyperglycemic hormone $(\mathrm{CHH})$ regulates the ammonia excretion and metabolism in white shrimp, Litopenaeus vannamei under ammonia-N stress. Sci. Total Environ. 723:138128. doi: 10.1016/j.scitotenv.2020.138128

Zmora, N., and Chung, J. S. (2014). A novel hormone is required for the development of reproductive phenotypes in adult female crabs. Endocrinology 155, 230-239. doi: 10.1210/en.20131603
Conflict of Interest: The authors declare that the research was conducted in the absence of any commercial or financial relationships that could be construed as a potential conflict of interest.

Copyright $\odot 2021 \mathrm{Liu}, \mathrm{Shi}, \mathrm{Ye}$, Liu and Zhu. This is an open-access article distributed under the terms of the Creative Commons Attribution License (CC BY). The use, distribution or reproduction in other forums is permitted, provided the original author(s) and the copyright owner(s) are credited and that the original publication in this journal is cited, in accordance with accepted academic practice. No use, distribution or reproduction is permitted which does not comply with these terms. 